\title{
Complications following acute severe haematogenous osteomyelitis of the long bones in children
}

\author{
Horn $\mathrm{A}^{1} \stackrel{-1}{ }$, Wever $\mathrm{S}^{2}$ (D), Hoffman $\mathrm{EB}^{3}$ \\ 1 MBChB(Pret), FC Orth (SA), MMed (UCT) Ortho; Consultant, Orthopaedic Surgery, Red Cross War Memorial Children's Hospital, Department of \\ Orthopaedic Surgery, University of Cape Town, South Africa \\ 2 MBChB(US); Registrar, Orthopaedic Surgery, Groote Schuur Hospital, University of Cape Town, Cape Town \\ ${ }_{3}$ MBChB (UCT), FCS Orth (SA); Associate professor (retired), Red Cross War Memorial Children's Hospital, Cape Town; University of Cape Town
}

Corresponding author: Dr Anria Horn, Postnet Suite 342, Pvt Bag X18, Rondebosch, 7701, South Africa; tel: +27 (0)21 4045108 ;

fax: +27 (0)21 447 2709; email: anriahorn@gmail.com

\begin{abstract}
Background: Acute haematogenous osteomyelitis $(\mathrm{AHO})$ of the long bones is frequently complicated by the development of sequestrum, pathological fracture, chronic osteomyelitis and growth arrest. We aimed to determine the frequency of complications and outcomes of patients with $\mathrm{AHO}$ of the long bones requiring surgical evacuation of pus. We furthermore examined the predictive value of clinical, biochemical and radiological variables on the development of complications following AHO.

Methods: Eighty-one patients were included. Clinical course, occurrence of complications and eventual outcome were documented. Complications were defined as: development of sequestrum, chronic osteomyelitis, pathological fracture and/or growth arrest. Results were analysed to determine factors predisposing to complications, and the difference in outcome between patients that developed complications and those that did not.

Results: The mean age at presentation was 7.5 years, and the mean follow-up was 1.8 years. Thirty-nine patients (48\%) developed 67 complications. Twenty-six patients developed sequestrum, 19 pathological fractures, 13 chronic osteomyelitis and nine growth arrest. No presenting feature was associated with an increased risk of developing complications. The percentage of cortex with radiological changes at 6 weeks $(66.1 \%$ vs $44.9 \%, p<0.0001)$ was associated with the development of complications. Duration of admission was significantly longer for those patients that developed complications $(21$ weeks vs 6 weeks; $p<0.001)$ Thirteen patients that developed complications had a poor outcome, compared to 0 patients that did not $(p<0.001)$.
\end{abstract}

Conclusion: AHO may result in severe disability, despite early recognition and adequate treatment. Early recognition of extensive bony involvement warrants increased vigilance to identify and manage complications timeously.

Level of evidence: Level 4

Key words: osteomyelitis, paediatric infection, complications, sequestrum, pathological fracture

Citation: Horn A, Wever S, Hoffman EB. Complications following acute severe haematogenous osteomyelitis of the long bones in children. SA Orthop J 2019;18(3):23-29. http://dx.doi.org/10.17159/2309-8309/2019/v18n3a1

Editor: Dr G Firth, University of the Witwatersrand, Johannesburg, South Africa

Received: January 2019

Accepted: April 2019

Published: August 2019

Copyright: $\odot 2019$ Horn A, et al. This is an open-access article distributed under the terms of the Creative Commons Attribution Licence, which permits unrestricted use, distribution and reproduction in any medium, provided the original author and source are credited.

Funding: No external funding was received.

Conflict of interest: None of the authors have any conflict of interest to declare. 


\section{Introduction}

Acute haematogenous osteomyelitis (AHO) is a common and serious disease affecting mostly young children. ${ }^{1}$ The incidence ranges from $1 / 800$ to $1 / 5000$, being higher in developing communities. $^{2}$ The exact incidence in South Africa is unknown. Mortality associated with AHO has decreased from $50 \%$ to $<1 \%$ since the antibiotic era and is usually a result of severe disseminated staphylococcal septicaemia and multi-organ failure. ${ }^{3}$ Morbidity remains high due to complications such as development of chronic osteomyelitis, pathological fracture and pseudo-arthrosis, bone loss with structural defects and growth arrest resulting in limb length discrepancy and angular deformity. A complication rate of $9-60 \%$ following $\mathrm{AHO}$ has been quoted in the South African literature. ${ }^{4-6}$ The sites most commonly affected are, in descending order, the femur, tibia, pelvis and humerus. ${ }^{7}$ Higher morbidity is associated with involvement of the long bones as cortical bone is more likely to develop pathological fracture, and growth arrest is associated with more severe deformity and disability than in the cancellous round and short bones of the foot, hand and axial skeleton. Factors reported to be associated with worse outcomes following $\mathrm{AHO}$ are delayed presentation, resistant infectious organisms, increased virulence of the infective organism, increased C-reactive protein (CRP) levels at presentation, ${ }^{8}$ immune compromise and other comorbidities.

Fifty to 60 children are admitted and treated for $\mathrm{AHO}$ at our institution every year, ${ }^{4}$ of whom $60-79 \%$ will have extraosseous pus collections. ${ }^{9}$ The purpose of this study is to evaluate the complications following $\mathrm{AHO}$ with extraosseous pus collections requiring surgical drainage. We also seek to determine whether certain presenting and radiological features are predictive of the development of complications and worse outcomes in these patients.

\section{Methods}

A retrospective review of case notes and X-rays was performed for patients who were admitted to our long-stay paediatric orthopaedic facility following incision and drainage for acute haematogenous osteomyelitis of the long bones from 2005 to 2016. Our local database was searched for the key words 'osteomyelitis', 'osteitis', 'infection' and 'sequestrum'. Only surgically managed patients were included. Those who were initially treated elsewhere and with incomplete data were excluded. Data was collected regarding duration of symptoms prior to presentation, clinical and biochemical findings at presentation, surgical findings, treatment course, causative organism, the occurrence of complications, the management thereof, and the eventual outcome. Complications were defined as 1) the development of sequestrum and/or chronic osteomyelitis; 2) pathological fracture and 3) growth arrest. X-rays of the affected long bone were routinely performed at time of presentation and six weeks after surgery. X-rays upon presentation were typically normal. X-rays taken at six weeks were analysed to determine the amount of diaphysis involved. The diaphysis was measured from proximal physis to distal physis. The anteroposterior (AP) and lateral projections were examined, and the percentage of involvement measured, added up and divided by 2 to arrive at the combined percentage (Figure 1).

In our study, poor outcome is defined as loss of joint mobility, limb length discrepancy $>2 \mathrm{~cm}$ and persistent chronic osteomyelitis.

Special investigations performed at presentation were blood cultures, a full blood count (FBC), erythrocyte sedimentation rate (ESR) and C-reactive protein (CRP).

Patients with a diagnosis of $\mathrm{AHO}$ undergo incision and drainage if there is clinically or radiologically evident drainable collections. In
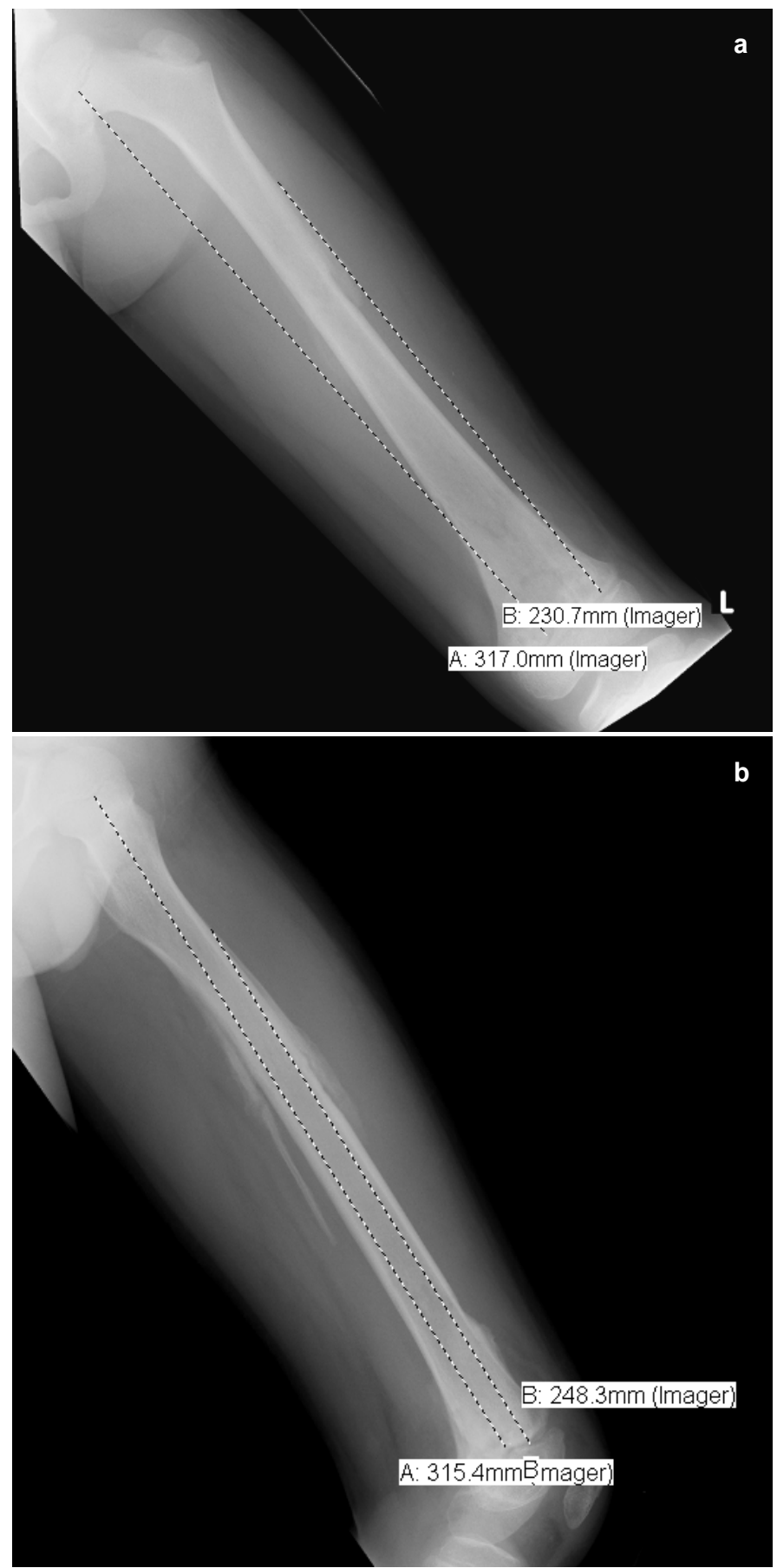

Figure 1. The method of calculating the percentage of diaphysis with radiographic changes

a). On the AP projection the length of the diaphysis is measured $(317 \mathrm{~mm})$ and the length of diaphysis with evident bony changes is measured (230.7 mm). $230.7 / 317=73 \%$.

b). On the lateral projection, the length of the diaphysis is measured $(315.4 \mathrm{~mm}$ ) and the length of the diaphysis with evident bony changes is measured $(248.3 \mathrm{~mm}) .248 .3 / 315.4=79 \%$. $(79+73) / 2=76$. Therefore $76 \%$ of the diaphysis is involved.

the case of clinical uncertainty, further imaging in the form of bone scan, ultrasound or MRI is requested to confirm the diagnosis and rule out the presence of a drainable collection.

Intra-operative specimens were routinely sent for microscopy, culture and sensitivity.

Results were analysed using SPSS ${ }^{\circ}$ (IBM Corp, 2017) to determine factors predisposing to the development of complications 
Table I: Features of and comparison between those patients that developed complications and those that did not

\begin{tabular}{|c|c|c|c|c|}
\hline & All patients & Complications & No complications & p-value \\
\hline & \multirow[t]{2}{*}{$N=81$} & $39(48 \%)$ & 42 (52\%) & \\
\hline & & \multicolumn{3}{|c|}{ Mean value (range) } \\
\hline Age (years) & $7.5(0.1-13.5)$ & $6.8(0.1-11.6)$ & $8.1(1.8-13.5)$ & 0.15 \\
\hline History of trauma (\%) & 46 & 53 & 40 & 0.38 \\
\hline Duration of symptoms (days) & $5(1-42)$ & $6(1-42)$ & $5(1-21)$ & 0.82 \\
\hline Temp $\left({ }^{\circ} \mathrm{C}\right)$ & $38(36-40.2)$ & $38.1(36-40)$ & $37.9(36.2-40.2)$ & 0.40 \\
\hline $\mathrm{CRP}(\mathrm{mmol} / \mathrm{dl})$ & $223.6(37.2-436)$ & $237.8(60-404)$ & $211.4(37.2-436)$ & 0.11 \\
\hline $\mathrm{ESR}(\mathrm{mm} / \mathrm{Hr})$ & $85.7(9.5-188)$ & $91.7(21-150)$ & $80.8(9.5-188)$ & 0.08 \\
\hline WCC (g/dl) & $17.0(1.3-36.1)$ & $16.9(1.3-36.1)$ & $17(5-28.8)$ & 0.31 \\
\hline $\begin{array}{l}\text { Duration of symptoms prior to } \\
\text { presentation (days) }\end{array}$ & $5(1-42)$ & $6(1-42)$ & $5(1-21)$ & 0.131 \\
\hline
\end{tabular}

Table II: Post-admission variables and comparison between patients that developed complications and those that did not

\begin{tabular}{|l|c|c|c|}
\hline \multicolumn{1}{|c|}{ Post-admission variable } & All patients & Complications & No complications \\
\hline & & Mean value (range) \\
\hline$>1$ theatre episode in acute phase & 24 & 13 & 11 \\
\hline Percentage bone involved at $6 / 52$ & $50.73(0-100)$ & $66.13(5-100)$ & 0.39 \\
\hline Time spent in hospital (weeks) & $12.9(2-65)$ & $21(3-65)$ & $6(0-100)$ \\
\hline Initial course of antibiotics (weeks) & $11.2(2-65)$ & $21(3-65)$ & $6(2-12)$ \\
\hline Poor outcome & 13 & 13 & 0.0001 \\
\hline
\end{tabular}

and worse outcomes following surgery for AHO. For parametric data, the student t-test and chi-square test was performed, for nonparametric data the Mann-Whitney $U$ test and the Fisher exact test were used. Statistical significance was set at $p \leq 0.05$. Multivariate analysis was also performed for the tested variables.

\section{Results}

A total of 103 patients were identified from the database. Of these, 14 were excluded on the grounds of initially being managed elsewhere. Six patients had incomplete data or were lost to followup and two are still actively being managed for the sequelae of acute osteomyelitis. Included in this review were 81 patients with 84 infected long bones. Five patients had multifocal osteomyelitis including some bones of the hands, feet and axial skeleton.

There were 59 boys and 22 girls (male:female 2.7:1) The mean age at presentation was 7.5 years (range $0.1-13.5$ ). The affected long bones were, in order of frequency, the tibia (41) the femur (35), the radius and ulna (5) and the humerus (3). The presenting features of the two groups, those with or without complications, are represented in Table I.

Blood culture results were available in $38(47 \%)$ of our patients. Thirty-two patients had blood cultures positive for methicillin sensitive Staphylococcus aureus (MSSA). Methicillin resistant Staphylococcus aureus (MRSA) was cultured in two patients only, skin commensals were cultured in one patient and three cultures were negative.

None of our patients were known to be HIV positive. Patients were not routinely tested for HIV, which is a limitation of this study.

Twelve patients had associated septic arthritis, of whom eight $(67 \%)$ developed complications. Of the 69 patients that did not have associated septic arthritis, 31 (45\%) developed complications. This was not statistically significant.

Four patients developed disseminated staphylococcal septicaemia and required ICU admission for associated problems such as pneumonia, empyema and multi-organ failure. Of these four patients, three developed complications.

Based on intra-operative samples, MSSA was the causative organism in 75 (93\%) patients. Two of these patients were coinfected with Escherichia coli and Klebsiella pneumonia respectively. The following organisms were cultured in one patient each: MRSA, Enterococcus faecalis, Klebsiella pneumonia, Staphylococcus epidermidis and Streptococcus pyogenes. In two patients tissue cultures were negative.

The post-operative variables of the two groups are represented in Table II.

Thirty-nine patients developed 67 complications after a mean of six months (1-36 months). Twenty-six developed sequestrum, 19 suffered pathological fractures, 13 developed chronic osteomyelitis and nine developed growth arrest. Of the 26 patients that developed sequestrum, 12 had pathological fractures and nine developed chronic osteomyelitis (Figure 2).

Twenty-seven of the 39 patients that developed complications required one or more surgical episode to address these complications. Fourteen patients required sequestrectomy, of whom two required femoral head excision for chronic osteomyelitis and avascular necrosis (AVN). Six patients required sequestrectomy and structural bone graft followed by prolonged immobilisation in an external fixator. Five patients had a hip spica applied for a femoral fracture and one patient each had a debridement, a high tibial osteotomy for deformity and a manipulation under anaesthesia.

Thirteen of the 39 patients that developed complications had a poor outcome. Four patients developed chronic osteomyelitis resistant to medical and surgical treatment (Figure 3). Four patients developed AVN of the hip, two of whom required a proximal femorectomy for source control and were left with a significant LLD and an unstable hip (Figure 4). Three patients suffered growth arrest and a resulting LLD $>2 \mathrm{~cm}$, and two patients developed AVN of the radial head and a stiff elbow (Figure 5). No patient that did not develop complications, as defined previously, had a poor outcome.

None of the pre-admission variables were significantly 

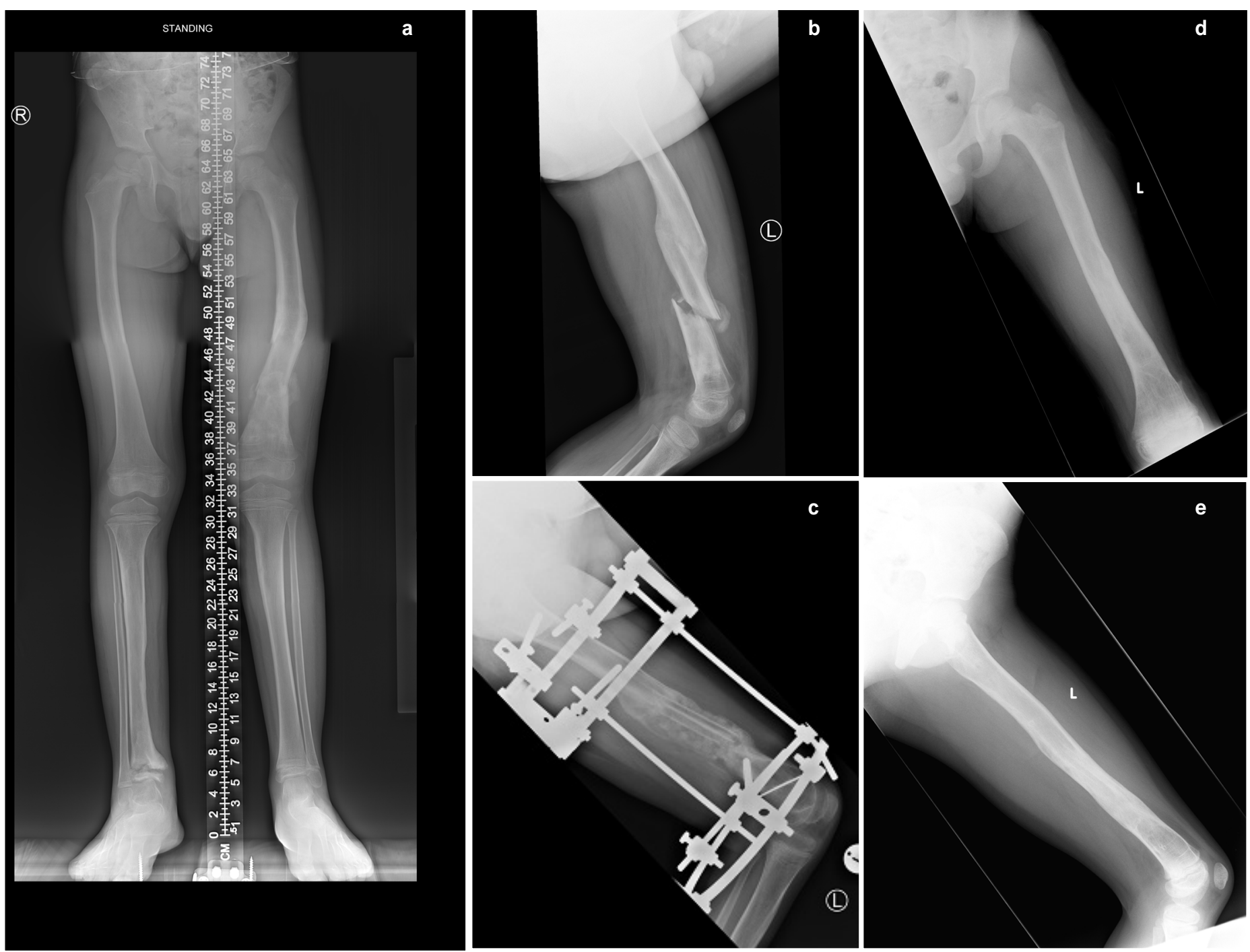

Figure 2. An 8-year old boy, one year following disseminated staphylococcal septicaemia. During the acute admission the right femur and left distal tibia were involved.

a). Leg length views showing united fracture of the proximal third of the femur with a new fracture in this distal third of the left femur. Physeal arrest is evident in the distal tibia on the right.

b). Lateral view of the left femur showing proximal femur united fracture and distal femur new fracture with callus. Sclerotic bone in the mid and distal diaphysis is suggestive of sequestrum.

c). Lateral view of the femur one month following autogenous bone grafting with non-vascularised fibula and iliac crest bone graft

$\mathrm{d}, \mathrm{e})$. AP and lateral projections of the left femur 14 months following

sequestrectomy and bone grafting. The bone graft has incorporated fully and there is no residual deformity.

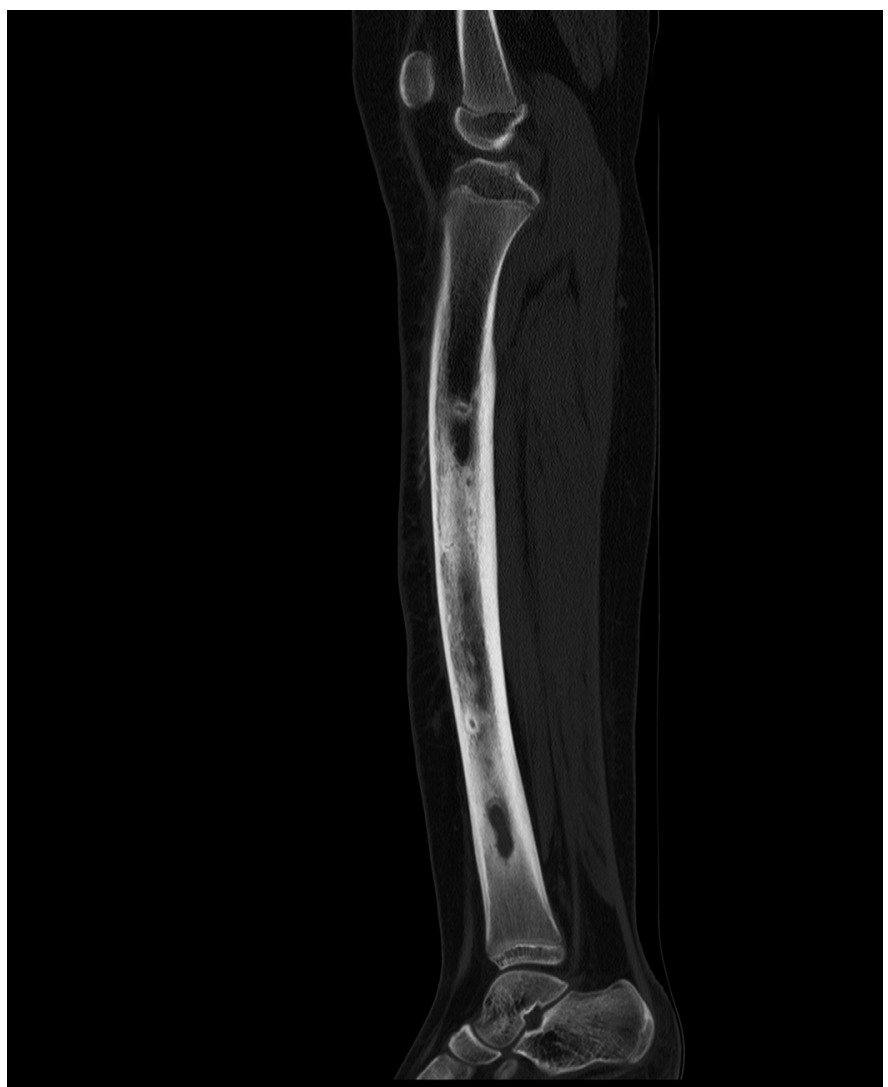




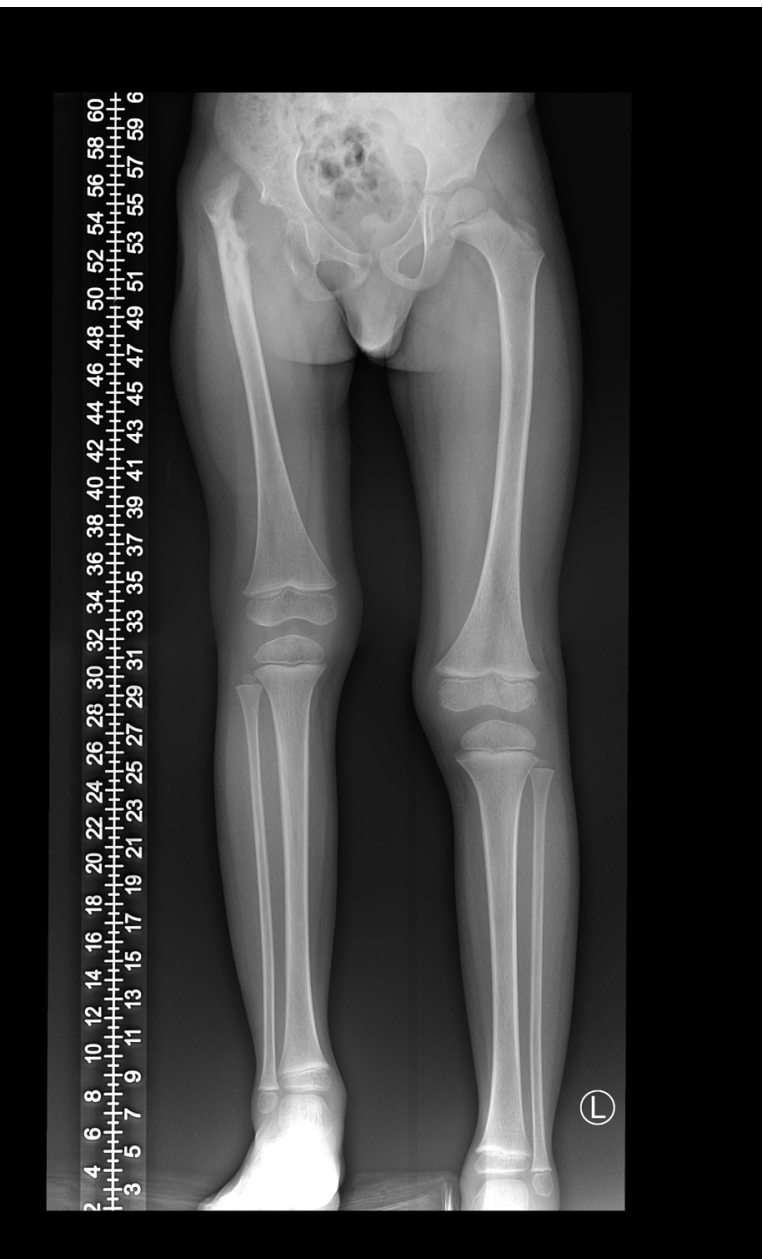

Figure 4. Leg length views of a 2-year old boy who developed AHO of the right femur with associated septic arthritis of the right hip as an infant. He has developed AVN of the right hip resulting in an unstable hip joint and a significant leg length discrepancy.

related to the development of complications. Univariate analysis indicated that the patient's age may be significant; however, in the multivariate analysis, this was not confirmed $(p=0.15)$.

Twenty-four patients required one or more repeat surgeries during the index admission. This consisted mostly of repeat washouts and debridements as indicated by recollection of pus or lack of clinical improvement. The need for repeat surgery during the acute admission was not associated with the development of complications.

The percentage of shaft involvement on X-ray at six weeks was significantly associated with the development of complications $(p=0.00041)$. Using a ROC calculation, a value of $45 \%$ shaft involvement on X-rays taken at six weeks was selected as an optimal cut-off point to determine likelihood of developing a complication. A total shaft involvement of $\geq 45 \%$ had a $78 \%$ sensitivity and $69.8 \%$ specificity for predicting the development of complications. The likelihood ratio for developing a complication with $\geq 45 \%$ shaft involvement was 2.58 (Cl 1.59-4.18).

The development of complications was, not surprisingly, associated with a longer initial course of antibiotics $(p<0.0001)$ and a longer total hospital admission time $(p<0.0001)$

\section{Discussion}

This study aims to highlight the complications and outcomes associated with osteomyelitis of the long bones in children where the disease is sufficiently advanced to require surgical

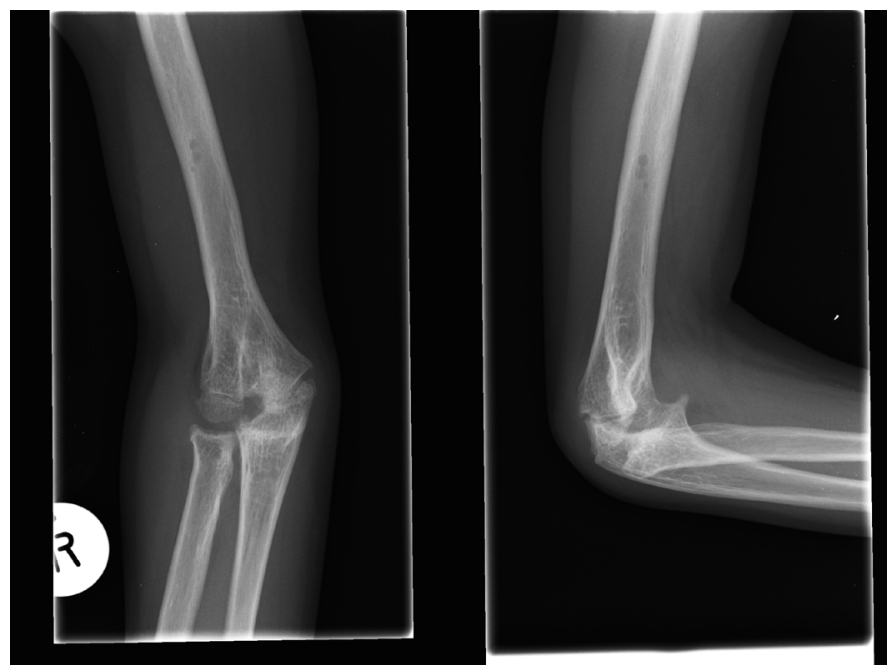

Figure 5. AP and lateral projections of the right elbow in a 6-year-old girl. She developed multifocal staphylococcal septicaemia two years prior with involvement of both hips, her left tibia and the right proximal ulna and elbow. X-rays demonstrate AVN of the trochlea and radial head with ankylosis of the elbow joint.

evacuation of extraosseous pus. This study population is by no means representative of all paediatric patients with osteoarticular infections and the findings of this study does not reflect the prognosis of patients with uncomplicated osteomyelitis requiring medical therapy only.

We did not find any presenting variable to be predictive of the development of complications. In a study from Costa Rica by Roine et al., ${ }^{8}$ the development of severe sequelae was associated with higher CRP values at admission, but this was not confirmed in our population. In accordance with our study, Roine et al. did not find that a delay in diagnosis nor the age of the patient to be predictive of the development of sequelae. These authors suggested that other factors, such as the virulence of the organisms and the patient's immune response, are the main determinants of outcome. UnkilaKallio et al., ${ }^{10}$ in a prospective study failed to prove a relationship between the levels of CRP, ESR and WCC at admission and the development of complications. In a study from Australia, Martin et al. determined that higher CRP levels at admission, as well as older age, upper limb involvement and indigenous status, were risk factors for complicated AHO. ${ }^{11}$

The CRP levels of our patients were on average higher than that of the reported literature. In the study by Martin et al., ${ }^{11}$ the mean CRP on admission for patients with complicated $\mathrm{AHO}$ was $95.5 \mathrm{mg} / \mathrm{l}(\mathrm{SD}=115.5)$ and in the study by Roine et al. ${ }^{8}$ it was $205 \mathrm{mg} / \mathrm{l}(\mathrm{SD}=89)$. In our cohort the mean for all patients was $223.6 \mathrm{mg} / \mathrm{l}(\mathrm{SD}=102.7)$. This may well indicate a more virulent organism or a more severe immune response as suggested by Roine.

In a systematic review by Ramachandran et $a .^{7}$ younger age and delay in diagnosis were associated with worse outcomes following AHO. In our study, there was a trend towards more complications in younger children, but this was found to be non-significant in multivariate analysis.

None of our patients were known to be HIV infected despite the high burden of HIV infection in South Africa. In an interesting study by Robertson et al. ${ }^{12}$ it was shown that patients infected with HIV had a predilection for osteoarticular infection caused by Streptococcus pneumoniae. This organism was not cultured in any of our patients. This may also be attributed to the institution of a national antistreptococcal vaccination programme in 2009.

Methicillin resistant Staph. aureus (MRSA) was identified as the causative organism in only two $(2.4 \%)$ of our patients. The 
prevalence of MRSA appears to be much lower in African countries than in developed countries. In a study performed in five major African cities, the incidence of MRSA among Staph. aureus isolates was $15 \%^{13}$ and in a recently published paper from a South African tertiary institution the incidence of MRSA was $15.3 \% .{ }^{14}$ Both these studies included all Staph. aureus isolates and not only osteoarticular infections. This is in stark contrast to the reported incidence of up to $88 \%$ in certain populations in the United States..$^{15,16}$ In another study by Arnold et al., ${ }^{17}$ also from the US, the incidence of MRSA as the causative organism for AHO rose from $4 \%$ to $40 \%$ during the study period of four years. Within our institution and across our referral base, the empiric treatment for osteoarticular infection consists of narrow spectrum specific antibiotics in the form of cloxacillin or flucloxacillin. Antibiotic regimens are only adjusted in the case of poor clinical response or in the case of a positive culture for a nonsensitive organism. Maintaining this strict antibiotic stewardship most probably contributes to the lower rate of community acquired MRSA in our patients.

Thirty-nine patients (48\%) developed 67 complications consisting of pathological fractures, chronic osteomyelitis with the formation of sequestrum, and growth arrest. There is great variability in the published literature as to what comprises complications following $\mathrm{AHO}$ and it is therefore difficult to compare our results. Belthur et al. reported a $4.7 \%$ pathological fracture rate following $\mathrm{AHO}$ in their population. ${ }^{16} \mathrm{All}$ their patients routinely underwent surgical drainage of intra- or extraosseous pus at initial presentation. Seven of the 19 patients that developed pathological fracture required surgery to address the fracture or complications associated therewith. Roine et al. defined severe sequelae as recurrent infection, pathological fracture or X-ray changes involving $>1 / 3$ of the shaft of the affected bone. ${ }^{8}$ They reported a $19 \%$ incidence of severe sequelae at twomonth follow-up. This follow-up period is probably too short to detect all complications following $\mathrm{AHO}$ as complications such as growth arrest became apparent as late as three years following presentation in our patients. In a study published by our institution 20 years ago, Broodryk et al. ${ }^{4}$ identified pathological fracture in $21 / 620$ patients (3.4\%). This low incidence compared to the recent study $(23 \%)$ can possibly be explained by our exclusion of simple osteomyelitis treated medically and infections not involving the long bones.

X-rays taken at the time of presentation seldom, if ever, demonstrate bony changes. We routinely take $X$-rays of the affected limb six weeks following the onset of disease. This is to quantify and qualify bony changes and identify sequestrum early. We found a statistically significant increased risk of developing complications with increased percentage of shaft involvement. To our knowledge, this is the first publication to describe this radiological feature as a risk factor for the development of complications. Belthur et al. analysed certain MRI features at presentation and its association with the development of pathological fracture. ${ }^{16}$ The finding of a sharp zone of marrow enhancement and the presence of an intramuscular abscess was significantly associated with pathological fracture. The cost and availability of MRI limits the use of this investigation to stratify risk and we only perform MRI in cases of clinical uncertainty.

The presence of sequestrum on the X-rays taken at six weeks was defined as a complication in our study and was in itself associated with the development of pathological fracture and chronic osteomyelitis. In a previously published paper, all patients that developed pathological fracture had structural sequestrum on X-ray. ${ }^{4}$

Despite early identification and optimal treatment of complications, $13 / 39$ patients (33\%) that developed complications had a poor outcome at final follow-up. AVN of the femoral head and proximal radius accounted for six of these, a devastating and irreversible complication.

Similar to other published series, ${ }^{8,16}$ the development of complications was associated with significantly prolonged periods of antibiotic treatment and hospitalisation. This naturally increases the economic and social burden of the disease but also has a negative psychological impact on the patient. Prolonged hospital admissions have been shown to predispose children to behavioural problems, delinquency and even unstable job patterns in adulthood. ${ }^{18}$

\section{Conclusion}

Acute haematogenous osteomyelitis remains an important cause of morbidity in children. No modifiable risk factors for developing complications were identified in this study. The percentage of bone involvement on X-ray taken six weeks following onset of disease was predictive of the development of complications, which may aid the surgeon in decision-making and prompt early intervention for the prevention or treatment of complications. The development of complications was associated with significantly prolonged hospital admission and antibiotic treatment, which increases the social and economic burden of disease.

There are several limitations to this study. It is a retrospective study and not all patients had the same investigations performed systematically. HIV testing was not routinely performed in our patients and we cannot, therefore, conclusively report on the interaction between $\mathrm{HI}$ infection and $\mathrm{AHO}$. Our method of selection naturally excludes all uncomplicated osteomyelitis, and our findings can therefore not be generalised to all cases of osteoarticular infection. Our institution only treats patients up to the age of 13 years, thereby excluding the older paediatric population.

\section{Ethics statement}

This study was approved by our institutional Human Research Ethics Committee (HREC 580/2017).

\section{Declaration}

The authors declare authorship of this article and that they have followed sound scientific research practice. This research is original and does not transgress plagiarism policies.

\section{Author contributions}

$\mathrm{AH}$ : Primary author

SW: Data collection and interpretation

EBH: Guidance and editing

\section{ORCID}

A Horn (iD http://orcid.org/0000-0002-4159-6520

S Wever (iD http://orcid.org/0000-0001-7072-1565

\section{References}

1. Blyth MJG, Kincaid R, Craigen M, Bennet G. The changing epidemiology of acute and subacute haematogenous osteomyelitis in children. Bone Joint J 2001;83(1):99-102.

2. Steer AC, Carapetis JR. Acute hematogenous osteomyelitis in children. Pediatr Drugs 2004;6(6):333-46.

3. Yeo A, Ramachandran M. Acute haematogenous osteomyelitis in children. BMJ 2014;348(315):66.

4. Broodryk AP SP, Hoffman EB. Fractures following acute haematogenous osteomyelitis in children. SA Bone Joint Surg. 1997;VII(4):20-2.

5. Nunn T, Cheung W, Rollinson P. A prospective study of pyogenic sepsis of the hip in childhood. Bone Joint J. 2007;89(1):100-6.

6. Hastings C, Hoffman E. Avascular necrosis following septic arthritis of the hip in children. J Bone Joint Surg [Br] 1995;77-B (Suppl 3):250 
7. Dartnell J, Ramachandran M, Katchburian M. Haematogenous acute and subacute paediatric osteomyelitis. J Bone Joint Surg [Br] 2012;94(5):584-95.

8. Roine I, Arguedas A, Faingezicht I, Rodriguez F. Early detection of sequela-prone osteomyelitis in children with use of simple clinical and laboratory criteria. Clin Infect Dis 1997;24(5):849-53.

9. Martin N, Ahmed H, Dix-Peek S, Hoffman E. Acute Osteomyelitis in Children: A 20 Year Study. Orthop Proc 2005;87-B (Supp III):279

10. Unkila-Kallio L, Kallio MJ, Peltola H, Eskola J. Serum C-reactive protein, erythrocyte sedimentation rate, and white blood cell count in acute hematogenous osteomyelitis of children. Pediatrics 1994;93(1):59-62.

11. Martin AC, Anderson D, Lucey J, Guttinger R, Jacoby PA, Mok TJ, et al. Predictors of outcome in pediatric osteomyelitis: Five years' experience in a single tertiary Center. Pediatr infect Dis $J$ 2016;35(4):387-91.

12. Robertson AJ, Firth GB, Truda C, Ramdass DA, Groome M, Madhi $\mathrm{S}$. Epidemiology of acute osteoarticular sepsis in a setting with a high prevalence of pediatric HIV infection. Journ Pediatr Orthop 2012;32(2):215-9.

13. Breurec $S$, Fall $C$, Pouillot R, Boisier P, Brisse $S$, Diene-Sarr F, et al. Epidemiology of methicillin-susceptible Staphylococcus aureus lineages in five major African towns: high prevalence of PantonValentine leukocidin genes. Clin Microbiol Infect 2011;17(4):633-9.

14. Oosthuysen W, Orth H, Lombard C, Sinha B, Wasserman E. Population structure analyses of Staphylococcus aureus at Tygerberg Hospital, South Africa, reveals a diverse population, a high prevalence of Panton-Valentine leukocidin genes, and unique local methicillin-resistant S. aureus clones. Clin Microbiol Infect 2014;20(7):652-59.

15. Martínez-Aguilar G, Avalos-Mishaan A, Hulten K, Hammerman W, Mason Jr EO, Kaplan SL. Community-acquired, methicillinresistant and methicillin-susceptible Staphylococcus aureus musculoskeletal infections in children. Pediatr infect Dis $J$ 2004;23(8):701-6.

16. Belthur MV, Birchansky SB, Verdugo AA, Mason Jr EO, Hulten KG, Kaplan SL, et al. Pathologic fractures in children with acute Staphylococcus aureus osteomyelitis. J Bone Joint Surg (Am) 2012;94(1):34-42.

17. Arnold SR, Elias D, Buckingham SC, Thomas ED, Novais E, Arkader $A$, et al. Changing patterns of acute hematogenous osteomyelitis and septic arthritis: emergence of communityassociated methicillin-resistant Staphylococcus aureus. Journ Pediatr Orthop 2006;26(6):703-708.

18. Bonn M. The effects of hospitalisation on children: a review. Curationis 1994;17(2):20-4. 